\title{
A Quantitative Lusin Theorem for Functions in $\mathrm{BV}$
}

\author{
András Telcs*, Vincenzo Vespri ${ }^{\dagger}$
}

November 19, 2013

\begin{abstract}
We extend to the BV case a measure theoretic lemma previously proved by DiBenedetto, Gianazza and Vespri $([1])$ in $W_{l o c}^{1,1}$. It states that if the set where $u$ is positive occupies a sizable portion of a open set $E$ then the set where $u$ is positive clusters about at least one point of $E$. In this note we follow the proof given in the Appendix of [3] so we are able to use only a 1 -dimensional Poincaré inequality.
\end{abstract}

\section{Introduction}

For $\rho>0$, denote by $K_{\rho}(y) \subset \mathbb{R}^{N}$ a cube of edge $\rho$ centered at $y$. If $y$ is the origin on $\mathbb{R}^{N}$, we write $K_{\rho}(0)=K_{\rho}$. For any measurable set $A \subset \mathbb{R}^{N}$, by $|A|$ we denote its $N$-dimensional Lebesgue measure.

*Department of Quantitative Methods, Faculty of Economics, University of Pannonia, Veszprém, Hungary \& Department of Computer Science and Information Theory, Budapest University of Technology and Economic, Magyar Tudósok Körútja 2, H-1117 Budapest, (HUNGARY), telcs.szit.bme@gmail.com

${ }^{\dagger}$ Dipartimento di Matematica ed Informatica Ulisse Dini, Universitá degli studi di Firenze Viale Morgagni, 67/a I-50134 Firenze (ITALIA) vincenzo.vespri@unifi.it. Member of GNAMPA (INdAM). 
If $u$ is a continuous function in a domain $E$ and $u\left(x_{0}\right)>0$ for a point $x_{0} \in E$ then there is a $r>0$ such that $u(x)>0$ in $K_{r}\left(x_{0}\right) \cap E$. If $u \in C^{1}$ then we can quantify $r$ in terms of the $C^{1}$ norm of $u$.

The Lusin Theorem says if $u$ is a measurable function in a bounded domain $E$, than for any $\varepsilon>0$ there is a continuous function $g$ such that $g=u$ in $E$ except in a small set $V \subset E$ such that $|V| \leq \varepsilon$.

In this note we want to generalize the previous property in the case of mesaurable functions. Very roughly speaking, we prove that if $u \in B V(E)$ and $u\left(x_{0}\right)>0$ for a point $x_{0} \in E$ than for any $\varepsilon>0$ there is a positive $r$, that can be quantitatively estimated in terms of $\varepsilon$ and the $B V$ norm of $u$, such that $u(x)>0$ for any $x \in K_{r}\left(x_{0}\right) \cap E$ except in a small set $V \subset E$ such that $|V| \leq \varepsilon\left|K_{r}\left(x_{0}\right)\right|$. Obviously we wil state a more precise result in the sequel.

Such kind of result has natural application in regularity theory for solutions to PDE's (see for instance the monography ([2]) for an overview). The first time it was proved in the Appendix of ([3]) in the case of $W^{1, p}(E)$. It was generalized in the case of $W^{1,1}(E)$ in $([1])$. Here we combine the proofs of ([3]) and ([1]) in order to generalize this result in BV spaces. Moreover in this note we use a proof based only on 1-dimensional Poincaré inequality. This approach could be useful in the case anisotropic operators where it is likely that will be necessary to develop a new approach tailored on the structure of the operator (a first step in this direction can be found in $([4])$ ).

We prove the following Measure Theoretical Lemma.

Lemma 1.1 Let $u \in B V\left(K_{\rho}\right)$ satisfy

$$
\|u\|_{B V\left(K_{\rho}\right)} \leq \gamma \rho^{N-1} \quad \text { and } \quad|[u>1]| \geq \alpha\left|K_{\rho}\right|
$$

for some $\gamma>0$ and $\alpha \in(0,1)$. Then, for every $\delta \in(0,1)$ and $0<\lambda<1$ there exist $x_{o} \in K_{\rho}$ and $\eta=\eta(\alpha, \delta, \gamma, \lambda, N) \in(0,1)$, such that

$$
\left|[u>\lambda] \cap K_{\eta \rho}\left(x_{o}\right)\right|>(1-\delta)\left|K_{\eta \rho}\left(x_{o}\right)\right| .
$$

Roughly speaking the Lemma asserts that if the set where $u$ is bounded away from zero occupies a sizable portion of $K_{\rho}$, then there exists at least one point $x_{o}$ and a neighborhood $K_{\eta \rho}\left(x_{o}\right)$ where $u$ remains large in a large portion of 
$K_{\eta \rho}\left(x_{o}\right)$. Thus the set where $u$ is positive clusters about at least one point of $K_{\rho}$.

In Section 2, we operate a suitable partition of $K_{\rho}$. In Section 3 we prove the result in the $\operatorname{case} N=2$ ( an analagous proof works for $N=1$. We consider more meaningful to prove the result in the less trivial case $N=2$ ). In Section 4 , by an induction argument, we extend the lemma to any dimension.

\section{Proof - A partition of the cube}

It suffices to establish the Lemma for $u$ continuous and $\rho=1$. For $n \in \mathbb{N}$ partition $K_{1}$ into $n^{N}$ cubes, with pairwise disjoint interior and each of edge $1 / n$. Divide these cubes into two finite subcollections $\mathbf{Q}^{+}$and $\mathbf{Q}^{-}$by

$$
\begin{array}{ll}
Q_{j} \in \mathbf{Q}^{+} & \Longleftrightarrow \quad\left|[u>1] \cap Q_{j}\right|>\frac{\alpha}{2}\left|Q_{j}\right| \\
Q_{i} \in \mathbf{Q}^{-} & \Longleftrightarrow \quad\left|[u>1] \cap Q_{i}\right| \leq \frac{\alpha}{2}\left|Q_{i}\right|
\end{array}
$$

and denote by $\#\left(\mathbf{Q}^{+}\right)$the number of cubes in $\mathbf{Q}^{+}$. By the assumption

$$
\sum_{Q_{j} \in \mathbf{Q}^{+}}\left|[u>1] \cap Q_{j}\right|+\sum_{Q_{i} \in \mathbf{Q}^{-}}\left|[u>1] \cap Q_{i}\right|>\alpha\left|K_{1}\right|=\alpha n^{N}|Q|
$$

where $|Q|$ is the common measure of the $Q_{l}$. From the definitions of the classes $\mathbf{Q}^{ \pm}$

$\alpha n^{N}<\sum_{Q_{j} \in \mathbf{Q}^{+}} \frac{\left|[u>1] \cap Q_{j}\right|}{\left|Q_{j}\right|}+\sum_{Q_{i} \in \mathbf{Q}^{-}} \frac{\left|[u>1] \cap Q_{i}\right|}{\left|Q_{i}\right|}<\#\left(\mathbf{Q}^{+}\right)+\frac{\alpha}{2}\left(n^{N}-\#\left(\mathbf{Q}^{+}\right)\right)$.

Therefore

$$
\#\left(\mathbf{Q}^{+}\right)>\frac{\alpha}{2-\alpha} n^{N}
$$

Consider now a subcollection $\overline{\mathbf{Q}}^{+}$of $\mathbf{Q}^{+}$. A cube $Q_{j}$ belongs to $\overline{\mathbf{Q}}^{+}$if $Q_{j} \in \mathbf{Q}^{+}$ and $\|u\|_{B V\left(Q_{j}\right)} \leq \frac{2 \alpha}{(2-\alpha) n^{N}}\|u\|_{B V\left(K_{1}\right)}$.

Clearly

$$
\#\left(\overline{\mathbf{Q}}^{+}\right)>\frac{\alpha}{2(2-\alpha)} n^{N}
$$


Fix $\delta, \lambda \in(0,1)$. The idea of the proof is that an alternative occurs. Either there is a cube $Q_{j} \in \overline{\mathbf{Q}}^{+}$such that there is a subcube $\tilde{Q} \subset Q_{j}$ where

$$
|[u>\lambda] \cap \tilde{Q}| \geq(1-\delta)|\tilde{Q}|
$$

or for any cube $Q_{j} \in \overline{\mathbf{Q}}^{+}$there exists a constant $c=c(\alpha, \delta, \gamma, \eta, N)$ such that

$$
\|u\|_{B V\left(Q_{j}\right)} \geq c(\alpha, \delta, \gamma, \lambda, N) \frac{1}{n^{N-1}}
$$

Hence if (2.2) does not hold for any cube $Q_{j} \in \overline{\mathbf{Q}}^{+}$, we can add (2.3) over all such $Q_{j}$. Therefore taking into account (2.1), we have

$$
\frac{\alpha}{2-\alpha} c(\alpha, \delta, \gamma, N) n \leq\|u\|_{B V\left(K_{1}\right)} \leq \gamma
$$

and for $n$ large enough this fact leads to an evident absurdum.

\section{Proof of the Lemma 1.1 when $N=2$}

The proof is quite similar to the one of appendix A.1 of ([3]) to which we refer the reader for more details. For sake of semplicity we will use the same notation of $([3])$.

Let $K_{\frac{1}{n}}\left(x_{o}, y_{o}\right) \in \overline{\mathbf{Q}}^{+}$. WLOG we may assume $\left(x_{o}, y_{o}\right)=(0,0)$. Assume that

$$
\begin{gathered}
\left|[u \leq \lambda] \cap K_{\frac{1}{n}}\right| \geq \delta\left|K_{\frac{1}{n}}\right| \quad \text { and } \quad\left|[u>1] \cap K_{\frac{1}{n}}\right|>\frac{\alpha}{2}\left|K_{\frac{1}{n}}\right| \\
\|u\|_{B V\left(K_{\frac{1}{n}}\right)} \leq \frac{2 \alpha}{(2-\alpha) n^{2}}\|u\|_{B V\left(K_{1}\right)} .
\end{gathered}
$$

Denote by $(x, y)$ the coordinates of $\mathbb{R}^{2}$ and, for $x \in\left(-\frac{1}{2 n}, \frac{1}{2 n}\right)$ let $\mathfrak{Y}(x)$ the cross section of the set $[u>1] \cap K_{\frac{1}{n}}$ with lines parallel to $y$-axis, through the abscissa $x$, i.e.

$$
\mathfrak{Y}(x) \equiv\left\{y \in\left(-\frac{1}{2 n}, \frac{1}{2 n}\right) \text { such that } u(x, y)>1\right\} .
$$


Therefore

$$
\left|[u>1] \cap K_{\frac{1}{n}}\right| \equiv \int_{-\frac{1}{2 n}}^{\frac{1}{2} n}|\mathfrak{Y}(x)| d x .
$$

Since, by (3.1), $\left|[u>1] \cap K_{\frac{1}{n}}\right|>\frac{\alpha}{2}\left|K_{\frac{1}{n}}\right|$,

there exists some $\tilde{x} \in\left(-\frac{1}{2 n}, \frac{1}{2 n}\right)$ such that

$$
|\mathfrak{Y}(\tilde{x})| \geq \frac{\alpha}{4 n} .
$$

Define

$$
A_{\tilde{x}} \equiv\left\{y \in \mathfrak{Y}(\tilde{x}) \text { such that } \exists x \in\left(-\frac{1}{2 n}, \frac{1}{2 n}\right) \text { such that } u(x, y) \leq \frac{(1+\lambda)}{2}\right\} .
$$

Note that for any $y \in A_{\tilde{x}}$ the variation along the $x$ direction is at least $\frac{(1-\lambda)}{2}$. If $\left|A_{\tilde{x}}\right| \geq \frac{\alpha}{8 n}$, we have that the BV norm of $u$ in $K_{\frac{1}{n}}$ is at least $\frac{\alpha(1-\lambda)}{16 n}$ and therefore (2.3) holds.

If $\left|A_{\tilde{x}}\right| \leq \frac{\alpha}{8 n}$, we have that there exists at least a $\tilde{y} \in \mathfrak{Y}(\tilde{x})$ such that $\mathrm{u}(\mathrm{x}, \tilde{y}) \geq \frac{(1+\lambda)}{2}$ for any $x \in\left(-\frac{1}{2 n}, \frac{1}{2 n}\right)$.

Define

$$
A_{\tilde{y}} \equiv\left\{x \in\left(-\frac{1}{2 n}, \frac{1}{2 n}\right) \text { such that } \exists y \in\left(-\frac{1}{2 n}, \frac{1}{2 n}\right) \text { such that } u(x, y) \leq \lambda\right\} .
$$

Note that for any $x \in A_{\tilde{y}}$ the variation along the $y$ direction is at least $\frac{(1-\lambda)}{2}$.

If $\left|A_{\tilde{y}}\right| \geq \frac{\delta}{n}$ we have that the BV norm of $u$ in $K_{\frac{1}{n}}$ is at least $\frac{\delta(1-\lambda)}{2 n}$ and therefore (2.3) holds.

If $\left|A_{\tilde{y}}\right| \leq \frac{\delta}{n}$ we have that $\left|[u>\lambda] \cap K_{\frac{1}{n}}\right| \geq(1-\delta)\left|K_{\frac{1}{n}}\right|$ and therefore $(2.2)$ holds.

Summarasing either (2.2) or (2.3) hold. Therefore the alternative occurs and the case $N=2$ is proved. 


\section{Proof of the Lemma 1.1 when $N>2$}

Assume that Lemma 1.1 is proved in the case $N=m$ and let us prove it when $N=m+1$.

Let $z$ a point of $\mathbb{R}^{m+1}$. To make to notation easier, write $z=(x, y)$ where $x \in \mathbb{R}$ and $y \in \mathbb{R}^{m}$.

Let $K_{\frac{1}{n}}(z) \in \overline{\mathbf{Q}}^{+}$. WLOG we may assume $z=(0,0)$. Assume that

$$
\begin{gathered}
\left|[u \leq \lambda] \cap K_{\frac{1}{n}}\right| \geq \delta\left|K_{\frac{1}{n}}\right| \quad \text { and } \quad\left|[u>1] \cap K_{\frac{1}{n}}\right|>\frac{\alpha}{2}\left|K_{\frac{1}{n}}\right| \\
\|u\|_{B V\left(K_{\frac{1}{n}}\right)} \leq \frac{2 \alpha}{(2-\alpha) n^{m+1}}\|u\|_{B V\left(K_{1}\right)}
\end{gathered}
$$

For any $x \in\left(-\frac{1}{2 n}, \frac{1}{2 n}\right)$ consider the $m$-dimensional cube centered in $(x, 0)$, orthogonal to the $x$-axis and with edge $\frac{1}{n}$ and denote this cube $\bar{K}_{\frac{1}{n}}(x)$. Define $\bar{A}$ as the set of the $x \in\left(-\frac{1}{2 n}, \frac{1}{2 n}\right)$ such that

$$
\left|[u>1] \cap \bar{K}_{\frac{1}{n}}(x)\right|>\frac{\alpha}{4}\left|\bar{K}_{\frac{1}{n}}(x)\right|
$$

and

$$
\|u\|_{B V\left(\bar{K}_{\frac{1}{n}}(x)\right)} \leq \frac{16}{(2-\alpha) n^{m}}\|u\|_{B V\left(K_{1}\right)} .
$$

It is possible to prove that

$$
|\bar{A}| \geq \frac{\alpha}{8 n}
$$

Let $\bar{x} \in \bar{A}$ and apply Lemma 1.1 to $\bar{K}_{\frac{1}{n}}(\bar{x})$ (we can do so because $\bar{K}_{\frac{1}{n}}(\bar{x})$ is a $m$-dimensional set).

So we get the existence of a constant $\eta_{0}>0$ and a point $y_{o} \in \bar{K}_{\frac{1}{n}}(\bar{x})$ such that if we define the set

$$
A \equiv\left\{(\bar{x}, y) \in \bar{K}_{\frac{\eta_{0}}{n}}\left(\bar{x}, y_{0}\right) \text { such that } u(\bar{x}, y) \geq \frac{(1+\lambda)}{2}\right\}
$$

where $\bar{K}_{\frac{\eta_{0}}{n}}\left(\bar{x}, y_{0}\right)$ denotes the $m$-dimensional cube of edge $\frac{\eta_{0}}{n}$, centered in $\left(\bar{x}, y_{0}\right)$ and orthogonal to the $x$-axis, we have

$$
|A| \geq\left(1-\frac{\delta}{2}\right)\left(\frac{\eta_{0}}{n}\right)^{m}
$$


Define

$$
B \equiv\left\{y \in A \text { such that } \exists x \in\left(-\frac{1}{2 n}, \frac{1}{2 n}\right) \text { such that } u(x, y) \leq \lambda\right\} .
$$

Note that for any $y \in B$ the variation along the $x$ direction is at least $\frac{(1-\lambda)}{2}$. If $|B| \geq \frac{\delta}{2}\left(\frac{\eta_{0}}{n}\right)^{m}$, we have that the BV norm of $u$ in $K_{\frac{1}{n}}$ is at least $\frac{\delta(1-\lambda)}{4}\left(\frac{\eta_{0}}{n}\right)^{m}$ and therefore (2.3) holds.

If $|B| \geq \frac{\delta}{2}\left(\frac{\eta_{0}}{n}\right)^{m}$, taking in account (4.3) we have that in the cylinder $\left(-\frac{1}{2 n}, \frac{1}{2 n}\right) \times \bar{K}_{\frac{\eta_{0}}{n}}\left(0, y_{0}\right)$ the measure of the set where $u(x, y) \geq \lambda$ is greater than $(1-\delta) \frac{\eta_{o}^{m}}{n^{m+1}}$. Therefore $(2.2)$ holds in a suitable subcube of $K_{\frac{1}{n}}$.

Summarasing either (2.2) or (2.3) hold. Therefore the alternative occurs and the case $N>2$ is proved.

Acknowledgments: This research was supported by the Italian-Hungarian executive project HU11MO10" NonLinear Diffusion Processes and Mathematical Modelling in Finance".

\section{References}

[1] E. DiBenedetto, U. Gianazza, V. Vespri, Local clustering of the non-zero set of functions in $W^{1,1}(E)$. Atti Accad. Naz. Lincei Cl. Sci. Mat. Appl., 9, (2006), 223-225.

[2] E. DiBenedetto, U. Gianazza, V. Vespri, Harnack's inequality for degenerate and singular parabolic equations Springer Monographs in Mathematics (2011).

[3] E. DiBenedetto, V. Vespri, On the singular equation $\beta(u)_{t}=\Delta u$. Arch. Rational Mech. Anal. 132 (1995), 247-309.

[4] F. G. Düzgün, P.Marcellini, V. Vespri, NonLinear Anal., 94 (2014), 133-141. 